\title{
Reducing pre-operative length of stay for enterocutaneous fistula repair with a multi-disciplinary approach
}

\author{
Mark Chamberlain, Rebecca Dwyer \\ University College London Hospitals NHS Foundation Trust, UK
}

\begin{abstract}
Pre-operative assessment of complex surgical patients can be a lengthy process, albeit essential to minimise complication rates. In a tertiary referral unit specialising in the surgical repair of entercutaneous fistulas, a baseline audit revealed an average in-patient length of stay of 30.1 days, mainly caused by poor co-ordination between specialities. After the introduction of a weekly multi-disciplinary team meeting and the formalisation of a patient pathway, this admission length was reduced to 5.7 days $(p<0.01)$, resulting in significant savings to the department.
\end{abstract}

\section{Problem}

The colorectal surgery unit at University College London Hospitals (UCLH) is a tertiary referral centre for intestinal failure (IF) and the surgical repair of enterocutaneous fistulas (ECFs). In 2013 there were approximately two new referrals every month, often made informally directly to the surgical consultants, with similar numbers of repairs undertaken monthly.

ECF patients are inherently a complex group, with multiple previous major abdominal surgeries including resections and revision anastomoses for inflammatory bowel disease or intra-abdominal sepsis. Co-morbidities, including poor nutritional state or concurrent sepsis, contribute to impaired wound healing and subsequent refistulation and mortality[1][2][3]. In order to optimise them preoperatively, it was standard practice for many patients to be transferred to UCLH for an in-patient assessment period comprising surgical consultant review, accurate fistula output measurement, comprehensive review of imaging, as well as a consultant anaesthetic review and input from the nutrition team (if appropriate) to enhance their current parenteral feeding regime. All of this was done with the aim of managing risk-factors and reducing the likelihood of post-operative complications.[4][5]

There was noted to be a lack of overall co-ordination for these patients, and little communication between clinical teams of different specialities, meaning that often these patients were transferred out of hours when beds became available. This led to the appropriate staff not being informed of the admission at handover, creating delays in assessment by the nutrition, surgical, and anaesthetic teams, longer in-patient length of stays, and subsequent cost to the department. Important information, such as scan results, were often not transferred with the patient, requiring duplication once at UCLH with the associated cost and time burden. Additionally, given the variable length of stay of these patients, there were delays in transferring them back to their referring hospitals prior to the operation date while waiting for a bed to become available.

The issue of unduly long inpatient length of stay was noticed, with many patients waiting several weeks to be seen by the different specialities, or awaiting a bed back at their referring hospital after their assessment period while awaiting their ECF repair. These delays and waits had a serious impact on bed pressures, with beds being unnecessarily occupied, in turn contributing towards the significant loss the service was operating at.

\section{Background}

There are currently two supraregional nationally-designated adult intestinal failure units in England: St Mark's in London and Salford Royal in Manchester. Both accept tertiary referrals from other acute trusts around the UK for the surgical management of ECFs. Gyorki et al published on the need for specialist multidisciplinary units,[6] but Murphy et al showed that ECF repair outcomes in regional centres with appropriate experience are comparable to those in national units,[7] with the need for a multidisciplinary approach again highlighted by Ravindran.[8]

A multidisciplinary surgical approach to patient care has been described by Counihan as allowing culture change and effective coordination of patients,[9] and the development of a defined patient pathway with buy-in from all team members has been shown to help reduce the length of stay.[10]

\section{Baseline measurement}

An audit of length of stay of patients admitted for assessment of their ECFs was undertaken to provide background information on the extent of the problem.

As there was no central database of IF patients at that time (or accurate clinical coding), data were retrospectively collected on an Excel spreadsheet from the electronic patient lists kept by the colorectal unit junior doctors. This collated information for any patient with a documented ECF on the referral, date of admission, and date of discharge, falling within a period of nine months March to October 2013 inclusive. 
Within the audited period, nine in-patient assessment episodes were identified. One was excluded as the patient required a prolonged ITU stay and eventually died in hospital. The remaining eight episodes had an average of 30.1 bed-days per admission. Two of the eight went on to have repairs within the same period. Of the 20 surgical repairs recorded to have been performed within the nine months, eight (40\%) did not require assessment admission prior to their surgery, four were operated on after an assessment period, and four were seen whilst an inpatient at UCLH under a different internal referring team. Of the eight separate assessment periods during the audit, four patients were transferred back to the referring hospital and four were discharged home. An additional outcome of the audit was that one patient who had been lost to follow-up after a clinic appointment had been cancelled was identified and an operation date booked.

Following discussion with the clinical and adminstration staff involved with assessment of IF patients, several roadblocks were identified leading to the excess length of stay in the ECF assessment patient cohort. Initially, there was no central referral point, with individual consultants accepting patients for transfer, with no named individual co-ordinating the process and championing their elective transfer to $\mathrm{UCLH}$, to avoid the often lengthy waits on the urgent transfer list. There were also difficulties in important information not being shared between the referring hospital and UCLH due to lack of a standardised referral form, and the lack of communication between teams at UCLH did not allow for forward planning and avoidance of duplication.

Patients also normally had expectations of being transferred for imminent surgical repair of their ECF, which led to inevitable loss of trust when informed about waiting periods. Finally, bed pressures at referring hospitals coupled with lack of willingness to readmit patients led to difficulties in transferring patients back to their local hospital to await their operation.

\section{Design}

Several interventions were devised in order to simplify and centralise the referral process for ECF assessment patients. A dedicated secure NHS.net email address was set up as the point of contact for all queries and referrals, which was accessible by all administration and clinical staff involved in the project. A service page for intestinal failure was created on the UCLH website where all queries could be directed to. This provides information for patients and referring teams, as well as a new mandatory referral form that requested the relevant information needed by the teams at UCLH, such as a full medical and surgical history, current nutrition regimen, microbiology and venous access routes, operation notes, and relevant radiology and investigations. The website also included a new patient information leaflet that could be given to patient before arrival, answering questions on what the assessment process involved.

An ideal length of admission of five nights, from Sunday until Friday, was decided upon at an initial stakeholder meeting, allowing formalisation of the in-patient assessment pathway with scheduled appointments in pre-assessment and imaging. Administrative support was included to facilitate communication between UCLH and the referring unit, and to highlight the possible need to accept the patient back after their assessment.

A weekly MDT was introduced every Friday morning, bringing together the surgical, nutrition, radiology, anaesthetic, and perioperative care teams, as well as the administrative staff managing the theatre diary and elective bed bookings. At this meeting, new referrals were discussed with correspondence to the person referring if they were accepted, or requests for further information if required. The planned TCl patients for the following week were also discussed, ensuring that all teams were ready for their arrival and that elective investigations had been booked in advance. All assessment patients currently in hospital were discussed in order to ensure all required information had been acquired, as well as that all teams were prepared to proceed with surgical repair as the most appropriate management option. Patients with operation dates were also included, ensuring that all administration such as postoperative HDU bookings had been made prior to their arrival.

\section{Strategy}

It was important to engage with the senior management team in order to get approval for process changes, administrative support and timetabled consultant PAs. Consultants also needed to be actively involved to ensure they embraced the new referral process and understood the importance of attending the weekly planning MDT. Through meetings with the hospital's service improvement team and their help in estimating the cost-savings possible, an initial stakeholder event was held for everyone involved, where the identified issues were addressed and buy-in confirmed. An action plan was drawn up and tasks delegated to teams in order to achieve the changes planned. Further smaller meetings were subsequently held regarding peri-operative care involvement, preoperative clinic scheduling, and website design.

\section{Post-measurement}

After implementation of the MDT, a second audit was carried out for the six-month period January to June 2014. The standard against which performance was assessed was for all suitable patients undergoing repair of an ECF to have a separate preceding fivenight inpatient assessment period admission.

Twenty-two patients were referred during the second audit period and deemed appropriate for surgical management. Twelve were admitted for a period as pre-operative assessment, three of whom were complicated by sepsis requiring a prolonged ITU admission. The remaining nine had an average length of stay of 5.7 bed-days, a five-fold reduction compared to the initial audit. Statistical analysis with the Mann-Whitney $U$ test returns a $p$ value $<0.01$ between audits groups.

Based on a figure of $£ 303$ per bed day provided by the Trust's commissioning team, this works out to an average cost-saving in bed-days alone to the department of $£ 7,393.20$ per patient; a potential annual saving of over $£ 177,000$ in total. Ten of the 22 
(45\%) were investigated in outpatients by the peri-operative physicians and so did not require an admission.

As reported above, the changes to the IF service have led to a reduced length of stay for assessment by coordinating the multidisciplinary process, and reduced the proportion of patients requiring in-patient assessment by formalising outpatient pathways regarding imaging and pre-operative optimisation, both of which have contributed towards substantial savings for the department.

\section{Lessons and limitations}

During the course of the project, we found it was important to seek agreement from all stakeholders, including management, which is more easily obtained when a clear cost-saving element can be demonstrated.

Further work is needed to ascertain whether there has been any change in the post-operative length of stay following the introduction of the patient pathway and MDT, and whether the time from referral to operation has reduced, with fewer pre-operative clinic attendances.

This quality improvement project is limited both by the small number of patients in each audit and their variable pre-morbid condition, as some patients required a much more intense optimisation period prior to surgery.

\section{Conclusion}

We started this project with the aim of reducing the pre-operative length of stay for assessment of complex surgical patients in a tertiary-referral centre. Through the instigation of a weekly multidisciplinary team meeting and a formalised patient pathway including referral process, we have demonstrated a reduction in length of stay from 30.1 to 5.7 days that would result in an annual saving of over $£ 177,000$ on bed-day costs alone.

This has proved the benefit of clear pathways when several different specialities are involved in patient care, and shown how MDT communication is influential in maximising productivity in complex cases.

\section{References}

1. Dudrick SJ, Maharaj AR, McKelvey AA. Artificial nutritional support in patients with gastrointestinal fistulas. World $\mathrm{J}$ Surg 1999 Jun;23(6):570-6.

2. Brenner M, Clayton JL, Tillou A, Hiatt JR, Cryer HG. Risk factors for recurrence after repair of enterocutaneous fistula. Arch Surg 2009 Jun;144(6):500-5.

3. Lynch AC, Delaney CP, Senagore AJ, Connor JT, Remzi $\mathrm{FH}$, Fazio VW. Clinical outcome and factors predictive of recurrence after enterocutaneous fistula surgery. Ann Surg 2004 Nov;240(5):825-31.

4. Owen RM, Love TP, Perez SD, Srinivasan JK, Sharma J, Pollock JD, Haack CI, Sweeney JF, Galloway JR. Definitive surgical treatment of enterocutaneous fistula: outcomes of a 23-year experience. JAMA Surg 2013 Feb;148(2):118-26.

5. Polk TM, Schwab CW. Metabolic and nutritional support of the enterocutaneous fistula patient: a three-phase approach. World J Surg 2012 Mar;36(3):524-33.

6. Gyorki DE, Brooks CE, Gett R, Woods RJ, Johnston M, Keck JO, Mackay JR, Heriot AG. Enterocutaneous fistula: a single-centre experience. ANZ J Surg 2010 Mar;80(3):178-81.

7. Murphy J, Hotouras A, Koers L, Bhan C, Glynn M, Chan $C L$. Establishing a regional enterocutaneous fistula service: the Royal London hospital experience. Int J Surg 2013;11(9):952-6.

8. Ravindran P, Ansari N, Young CJ, Solomon MJ. Definitive surgical closure of enterocutaneous fistula: outcome and factors predictive of increased postoperative morbidity. Colorectal Dis 2014 Mar;16(3):209-18.

9. Counihan T, Gary M, Lopez E, Tutela S, Ellrodt G, Glasener R. Surgical Multidisciplinary Rounds: An Effective Tool for Comprehensive Surgical Quality Improvement. Am J Med Qual 2014 Sep 10.

10. White CM, Statile AM, White DL, Elkeeb D, Tucker K, Herzog D, Warrick SD, Warrick DM, Hausfeld J, Schondelmeyer A, Schoettker PJ, Kiessling P, Farrell M, Kotagal U, Ryckman FC. Using quality improvement to optimise paediatric discharge efficiency. BMJ Qual Saf 2014 May;23(5):428-36.

\section{Declaration of interests}

Nothing to declare.

\section{Acknowledgements}

Esther Rainbow, assistant general manager - Gl surgery.

Tara Donnelly, Director, Quality, Efficiency and Productivity programme.

Jonathan McCullough, consultant colorectal surgeon.

Katie Randall, improvement facilitator, Quality, Efficiency and Productivity team. 